\title{
Application of Myoelectric Potential for Personal Mobility Vehicles
}

\author{
Kouki Moriyama ${ }^{\mathrm{a}}$, Keiji Matsumoto ${ }^{\mathrm{a} *}$

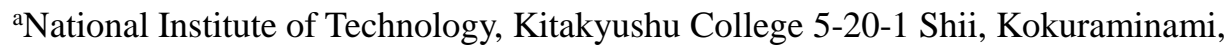 \\ Kitakyushu, Fukuoka, 802-0985, Japan \\ "Corresponding Author:kmatsu@kct.ac.jp
}

\begin{abstract}
Recently, several types of compact personal mobility vehicles (PMVs) have been developed. This study proposes a new type of PMV that can be used as a transportation device, not only for disabled persons, but for the elderly as well. The proposed PMV uses biological signals as a reference value. Myoelectric potential, owing to its ability to reflect human intention with a high probability, was selected from the various types of bioinformation. In this study, we design and fabricate a PMV and consider a feasible utilization method for myoelectric potential.
\end{abstract}

Keywords: PMV, myoelectric potential, control.

\section{Introduction}

In recent years, small personal mobility vehicles (PMVs) that differ from automobiles have received attention. Examples include the Winglet (manufactured by Toyota Motor Corporation) and Segway (a mobility vehicle individuals ride while standing that are operated by weight transfer) [1]. Ropits, developed by Hitachi, Ltd, is a mobility vehicle different from those mentioned above, in that individuals perform the driving operations from a driver's seat as in an ordinary car; however, Ropits differs from an ordinary car because it has an autonomous mobile function that relies on high-accuracy location estimation using sensors. Many PMVs have been researched and developed, such as lightweight PMVs, uniquely structured PMVs, and PMVs designed for particular missions [2-5]. Furthermore, because Japan is an aging society, PMVs for elderly or physically disabled persons have been developed. As PMVs that operate via weight transfer may be difficult to operate for individuals who have trouble walking, the PMV developed in this study uses a method that assists walking ability. This PMV contains an assisting system that estimates walking states by both acceleration and revolution sensors and drives a motor attached to a wheel.

A competition for disabled athletes who have enhanced abilities owing to robotics engineering technologies, called the Cybathlon, hosted by the Swiss National Center of Competence in Research Robotics, was recently held [7]. Myoelectric signals sent to the disabled body part are picked up and used to manipulate a machine playing a role of that body part. Although this is a significant attempt to support disabled persons via prosthetic devices with bioinformation, this is not directly associated with mobility devices, such as PMVs. S. Matugaura et al. reported an electric vehicle prototype that can support steering and braking for elderly people [8]. This prototype supports elderly people while they drive by estimating the operational state using not only a pedal and steering angle, but face temperature, electromyography (EMG), and heartbeat. Although an assisting robot caregiver using EMG and the system estimating the human state using EMG are reported [9-10], there is no report of a PMV that can be maneuvered using bioinformation.

In this study, we consider the possibility of a PMV driven using bioinformation and create a prototype. Myoelectric potential, owing to its ability to reflect human intention with a high probability, was selected in this study from the various types of bioinformation. We consider a control method using myoelectric potential of the left and right arms as the command value for the PMV instead of weight transfer. If the PMV we proposed can be realized, it will be one of the novel transportation devices in the future for disabled and elderly persons. In this study, the new PMV is designed and fabricated, and the control method using myoelectric potential is considered using an empirical approach. 


\section{Method}

\subsection{Structure of the fabricated PMV}

In this study, a standing-type PMV that can be easily designed was chosen because the ability to use EMG as the PMV control method was the study's focus. The proposed PMV, whose structure is not an inverted pendulum, has two driving wheels and two safety wheels, and the ability to drive at $2 \mathrm{~km} / \mathrm{h}$. The appearance and specifications of the fabricated PMV are shown in Fig.1 and Table.1, respectively. The base of the PMV is an aluminum plate with a thickness of $8 \mathrm{~mm}$ that has the ability to hold $80 \mathrm{~kg}$ of weight. A 50-W DC motor with gears directly mounted on the motor shaft are placed on each side of the PMV. Driving wheels $120 \mathrm{~mm}$ in diameter are placed on the left and right sides and connected to each other with an aluminum shaft $20 \mathrm{~mm}$ in diameter and are supported by left and right bearing units. Pulleys $30 \mathrm{~mm}$ in diameter are installed on both the back side of the tires and the gear shaft of the motors; the driving force from the motor to the tire is transmitted through a timing belt. Two lithium ion batteries of $7.2 \mathrm{~V}$ and 1.7 Ah (Tamiya Inc.) are used to provide power to the left and right sides of the motors.

Turning is performed by making a difference between the left and right sides of motor revolutions. Mechanical parts, such as the aluminum shaft or bearing unit, are installed on the back side of the PMV base, and the electric components, such as the motor drive circuit or battery, are mounted on the surface of the PMV base. To secure the riding area and protect the electrical components, a protective cover made of a transparent fiber-reinforced plastic material is placed over the PMV base surface. The designed control circuit for the PMV consists of the EMG sensors, a microcomputer, motor drive circuits, driving motors, and batteries (Fig.2). An AT-04-001 EMG sensor (Advancer Technologies Inc.) with an embedded electronic circuit that outputs the myoelectric potential that is filtered and amplified after rectification is used. The signal coming from the EMG sensor is captured in the Arduino through the $\mathrm{AD}$ converter. The Arduino produces the motor control signal after analyzing the driver's intention by processing the myoelectric potential. The output signals from the Arduino are isolated from the motor power source using photo-couplers to prevent malfunctions attributed to noise. The EMG generated by grip strength is used as a reference value for the motor. Left and right motors use the signal from the myoelectric potential sensors worn on the left and right arms. The signal processed inside the Arduino is transmitted to the motor drive circuit and operates the PMV.

Table.1 specification of fabricated PMV

\begin{tabular}{|c|c|}
\hline Size of PMV & $350[\mathrm{~mm}] \times 500[\mathrm{~mm}] \times 1340[\mathrm{~mm}]$ \\
\hline Diameter of wheel & $120[\mathrm{~mm}]$ \\
\hline Total mass & $13[\mathrm{~kg}]$ \\
\hline Maximum speed & $2[\mathrm{~km} / \mathrm{h}]$ \\
\hline Withstand load & $80[\mathrm{~kg}]$ \\
\hline Batteries & Li-ion battery $(7.2[\mathrm{~V}]) \times 2$ \\
\hline Actuator & $50[\mathrm{w}]$ DC motor $\mathrm{x} 2$ \\
\hline
\end{tabular}

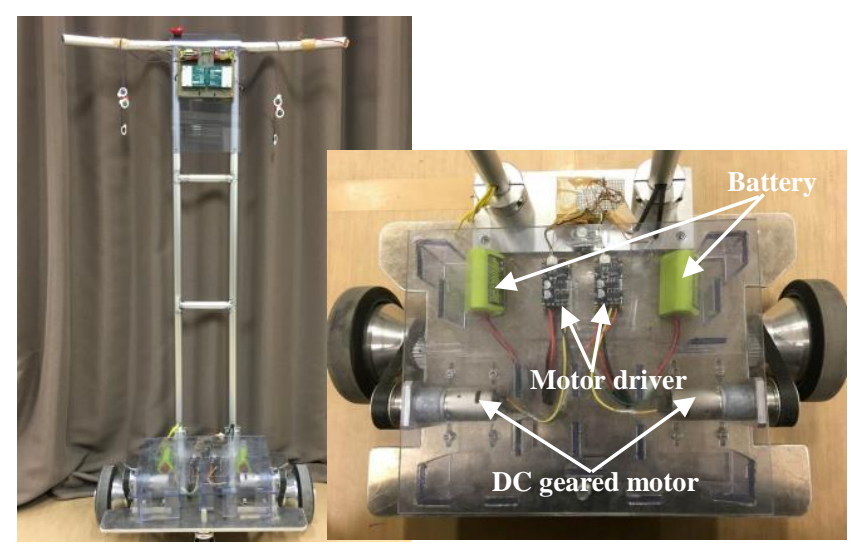

Fig.1 Appearance of fabricated PMV

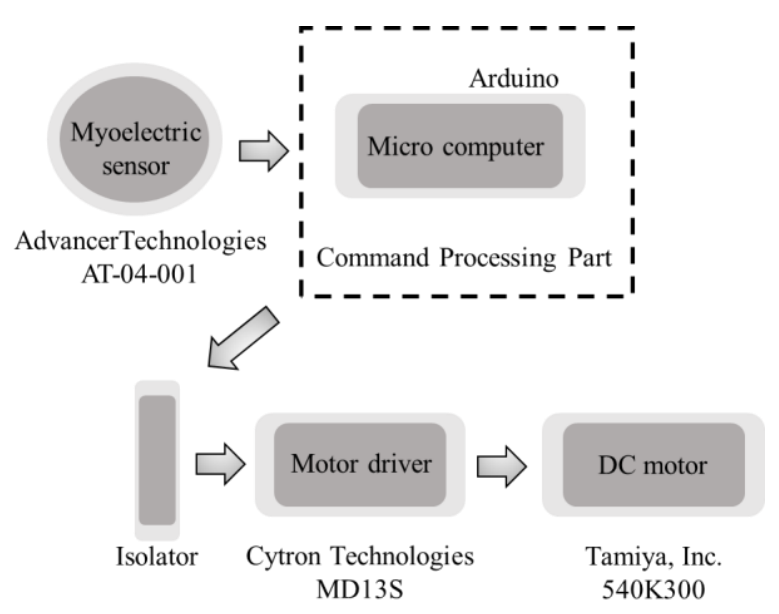

Fig. 2 configuration of the control system 


\subsection{Myoelectric potential}

When the brain activates muscles, the command signal is transmitted to the muscle fibers through motor nerves (Fig.3). In muscle fibers, action potentials are generated and propagated to the muscles. These action potentials are called EMGs. Surface EMG, which is a measurement method wherein electrodes are attached to the skin, is widely used for EMG measurement. As the mean potential of the surface EMG is approximately several microvolts to several millivolts, an amplifier is necessary for measurement [11]. Because the amplitude of EMG has the tendency to increase with muscle tension, there is a proportional relationship between EMG and muscle tension.

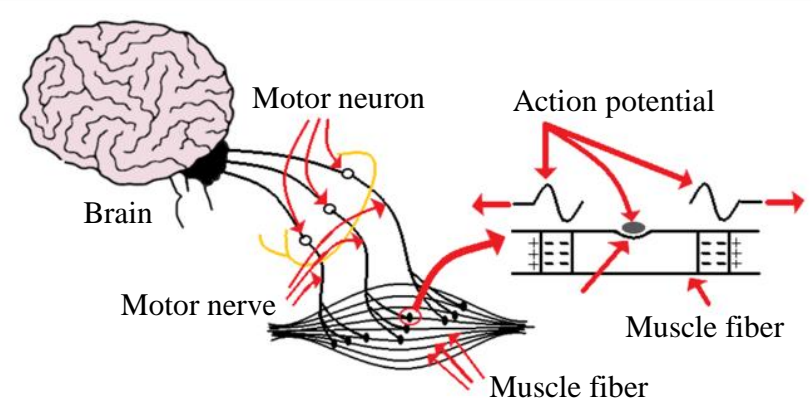

Fig.3 Mechanism of the myoelectric potential

\subsection{Muscle selection for controlling the PVM}

First, we investigated which myopotentials would be appropriate to manipulate the PMV. For usability, the deep digital flexor muscle and common digital extensor muscle were selected (Fig.4). Fig.5 shows the measurement results of the relationship between the grip strength and myoelectric potential for these muscles. The slopes of the approximately straight lines for the deep digital flexor muscle of the left and right arms were 0.090 and 0.085 , respectively. In the same way, the slopes of the approximately straight lines of the common digital extensor muscles of the left and right arms were 0.093 and 0.198 , respectively. The standard deviation of the deep digital flexor muscles of left and right arms were 0.347 and 0.210 , respectively, and that of the common digital extensor muscles of left and right arms were 0.228 and 0.219 , respectively. Although the electric potentials generated from grip strength fluctuate in all cases, the relationship can be thought of as a proportional relationship. Even so, the slopes of those data are different, even when comparing the same muscle in the left and right arms. Therefore, the system requires some type of compensable function, such as calibration, to employ grip strength as a reference value for motor control.

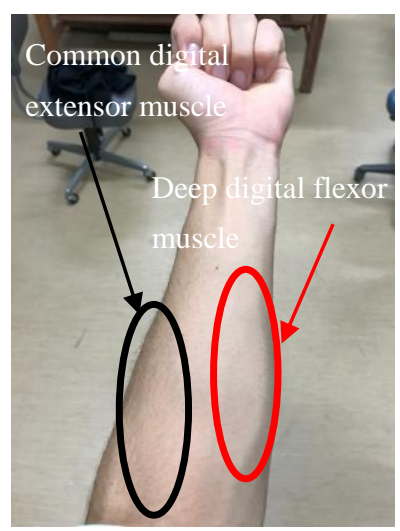

Fig.4 Positions of the muscle on an arm

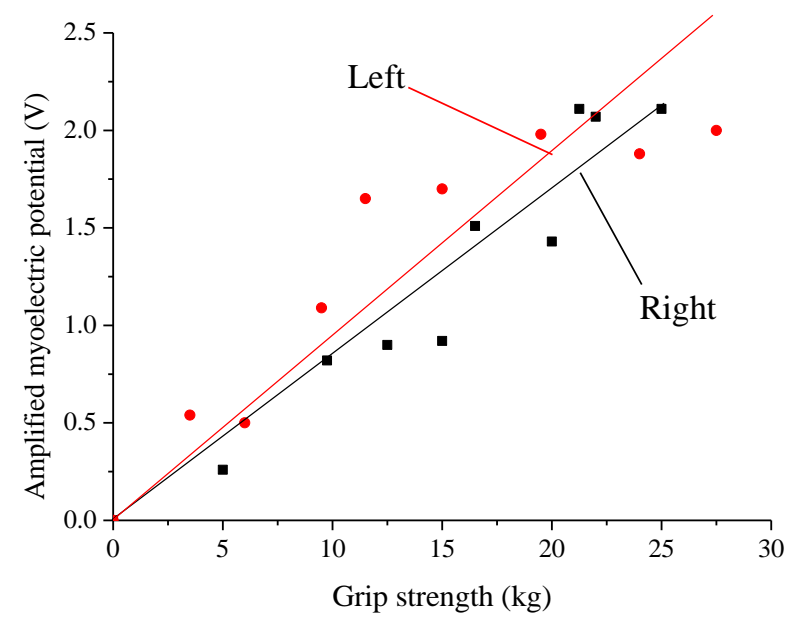

(1)

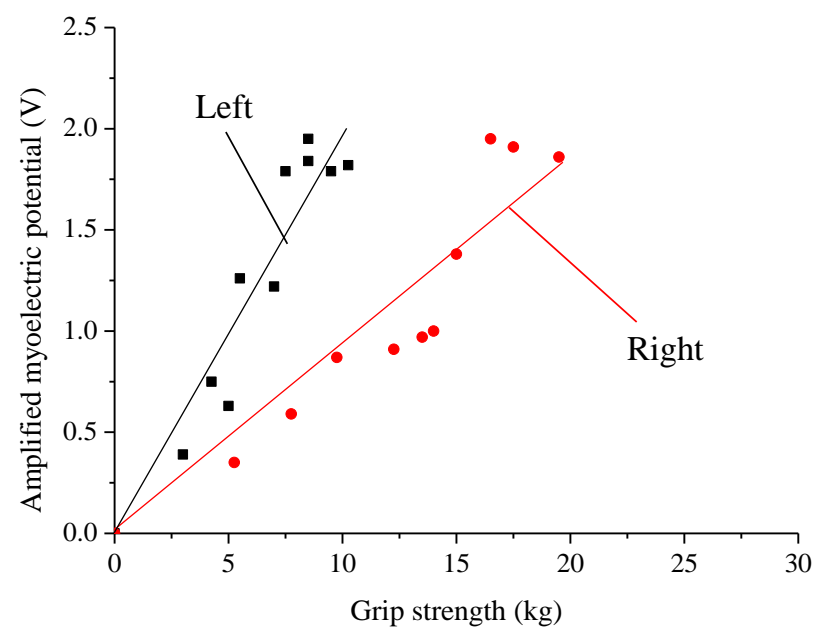

(2)

Fig.5 myoelectric potential associated with the grip strength

(1) Deep digital flexor muscle

(2) Common digital extensor muscle 


\subsection{Operating method using the myoelectric potential}

It is possible to control the PVM using grip strength because the generated myoelectric potential is approximately proportional to the grip strength. However, this method has a disadvantage in terms of controllability because a certain level of grip strength is required to generate the myoelectric potential necessary during operation. Considering the above, the operation procedure of the PMV was determined as follows:

1. Gripping for over $0.5 \mathrm{~s}$ is recognized as a stop command;

2. Gripping and loosening within $0.5 \mathrm{~s}$ is recognized as a start command.

According to the above procedure, moving forward, clockwise and counterclockwise turning, and stopping are possible. Fig.6 shows the myoelectric potentials under the two operational commands. To discriminate the operational commands more accurately, two threshold voltages, Th1 and Th2, were developed. This is because the system attempts to decrease the rate of false recognition due to noise by considering a hysteresis as a criterion for judgement. Th1 is the level to start identification of the input signal and Th2 is for the end mark of the input signal. When the input value exceeds Th1, the system starts observing from that point. Subsequently, if the input signal maintains a level higher than Th2 for $0.5 \mathrm{~s}$ or more, the system identifies it as the stop command and turns off the motor. If the input signal becomes lower than Th2 within $0.5 \mathrm{~s}$ after surpassing Th1, it determines that this signal is the start command and drives the motor. An emergency stop button was separately installed for safety.

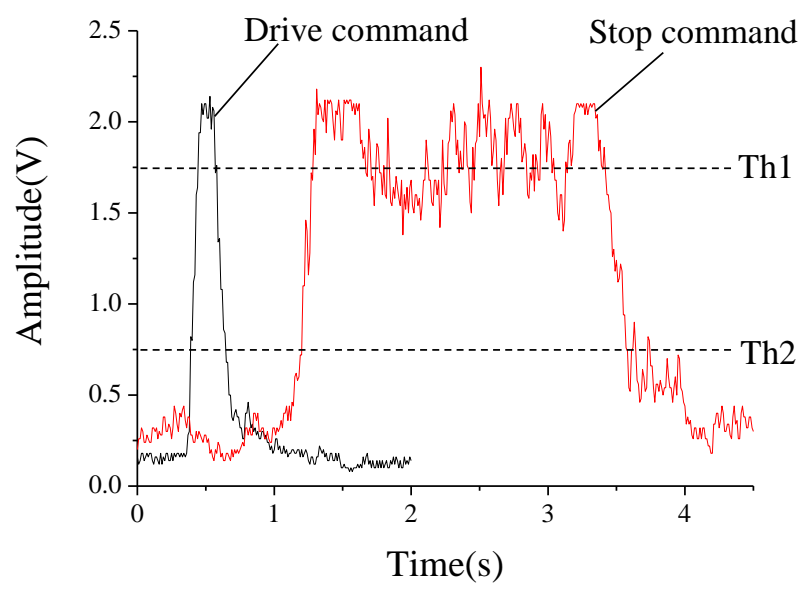

Fig.6 Myoelectric potentials of the operational commands

\section{Experimental results}

To evaluate the use of continuous operation using myoelectric potential commands, a simple test course, as shown in Fig.7, was prepared. This test course consisted of three 1.8-m straights, two 1.2-m straights, and four $90^{\circ}$ turns. PMV drivability can be tested by driving it along the test course. A G-MEN DR01 (SRIC.co) 3-axis acceleration sensor was used for this experiment. The experimental results are shown in Fig.8. Figs.8 (1) and (2) show the pitch acceleration generated when moving forward and the yaw acceleration generated when turning, respectively. The large values in the Fig. 8 indicate transitions from one motion to another.

It was found that the behavior of the PMV was intentionally changed because relatively large acceleration data were regularly plotted. However, as the operation details are difficult to understand from Fig.8, enlarged horizontal axes are shown in Fig.9, Fig.10, and Fig.11. These figures (1) show pitch acceleration; positive and negative values represent the generated acceleration of backward and forward motion, respectively. Figures (2) show yaw acceleration, and the positive and negative values represent the generated acceleration of the right and left sides, respectively. From straight 1 to straight 5, because backward acceleration was generated at the beginning of the forward movement and forward acceleration was generated at the end of the forward movement, it was found that the PMV went straight. At corner 1 and corner 4, because left-side acceleration was generated at the beginning of the movement and right-side acceleration was generated at the end of the movement, it was found that the PVM turned right.

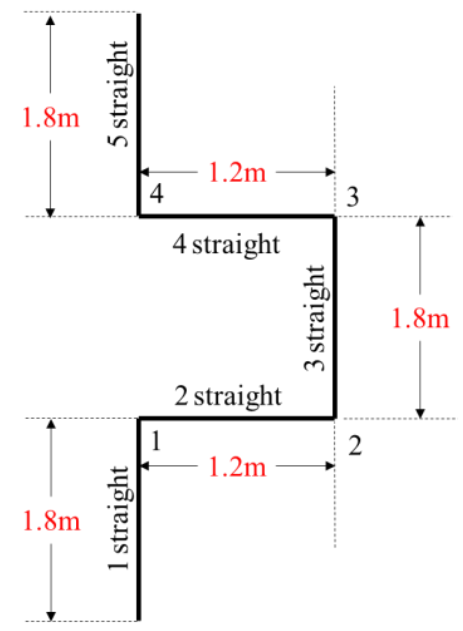

Fig.7 layout of a test course for the PMV experiment 

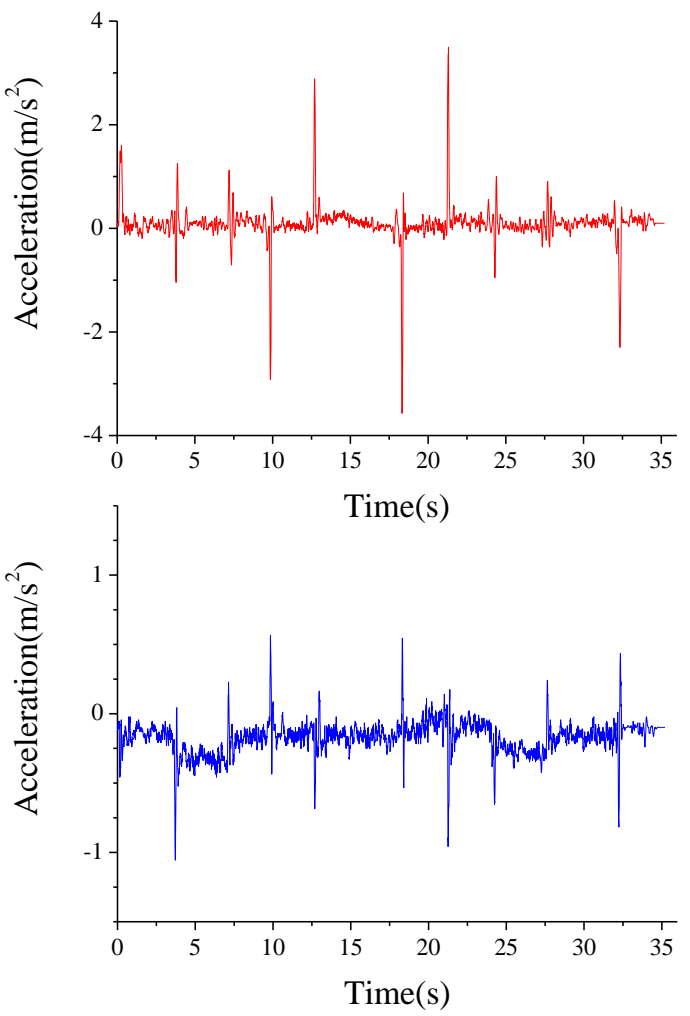

(2)

Fig.8 Acceleration data obtained from driving PMV on the test course (1) Pitch acceleration (2) yaw acceleration
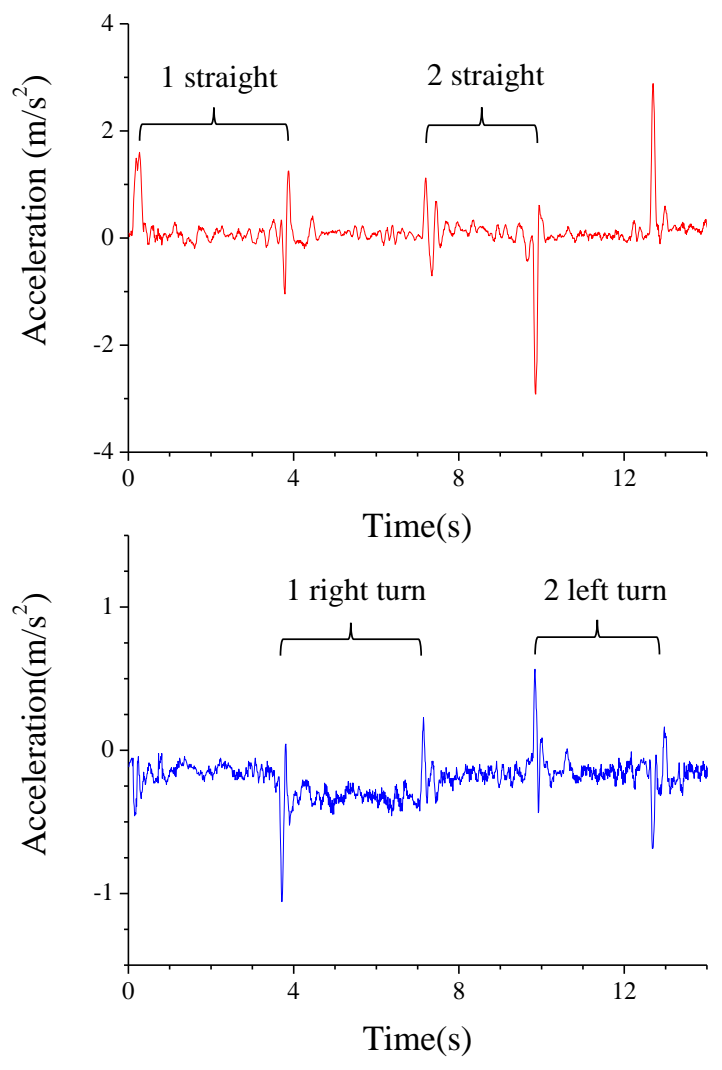

Fig.9 Detail of the measured acceleration (0 to 14 second) (1) Pitch acceleration (2) yaw acceleration
In the same way, at corner 2 and corner 3 , because right-side acceleration was generated at the beginning of the movement and left-side acceleration was generated at the end of the movement, it was found that the PVM turned left. It was thought that the PVM could be controlled according to the intention of the driver because these motions occurred along the test course. The times required for the four corners were $3.4 \mathrm{~s}, 2.9 \mathrm{~s}, 3.0 \mathrm{~s}$, and $3.3 \mathrm{~s}$, respectively; the average speed calculated was $3.2 \mathrm{~s}$.

Although cornering is a rotary motion, the speed was equivalent to approximately $0.88 \mathrm{~km} / \mathrm{h}$ when converted to a straight motion. Compared to the measured $1.38 \mathrm{~km} / \mathrm{h}$ straight speed, the cornering speed was $36 \%$ slower than the straight speed. The maximum difference among the times spent for corners was $0.5 \mathrm{~s}$ and the distance calculated by traveling at $0.88 \mathrm{~km} / \mathrm{h}$ was approximately $12.2 \mathrm{~cm}$. Although the slight difference in time was confirmed among the four corners, it is thought that the PMV was controlled in line with the driver's intention. The times spent for the $1.2-\mathrm{m}$ straights were $2.7 \mathrm{~s}$ and $3.0 \mathrm{~s}$; the distances calculated traveling at $1.38 \mathrm{~km} / \mathrm{h}$ would be $1.04 \mathrm{~m}$ and $1.15 \mathrm{~m}$, respectively. Thus, it was thought that the PMV could be operated on the two $1.2-\mathrm{m}$ straights. The times spent for the three $1.8-\mathrm{m}$ straights were $3.56 \mathrm{~s}, 5.6 \mathrm{~s}$, and $4.74 \mathrm{~s}$; the distances calculated traveling at $1.38 \mathrm{~km} / \mathrm{h}$ would be $1.36 \mathrm{~m}, 2.15 \mathrm{~m}$, and $1.82 \mathrm{~m}$, respectively.

Although the PMV traced the fifth straight, the traveled distances of the first and second straight were different from the course length. The reasons for not being able to trace the course are considered as follows. First, the initiation time of the myoelectric potential of the driver was not constant and the amplitude of the generated myoelectric potential was not stable. For this reason, the PMV sometimes responded with a slight delay from the driver's intention. Next, it is conceivable that a difference of several tens of centimeters occurs depending on the position of the PMV passing through the course because the size of the $\mathrm{PMV}$ is $350 \times 500 \mathrm{~mm}$. Last, the revolution speed of the DC motor changes with the unstable torque required owing to the condition of the course because the motor drive system does not use feedback control. These concerns should be considered and improved upon in the future. However, it was confirmed that the PMV could almost be controlled along with the driver's intentions. These experimental results imply the feasibility of a PMV operated using myoelectric potential. 

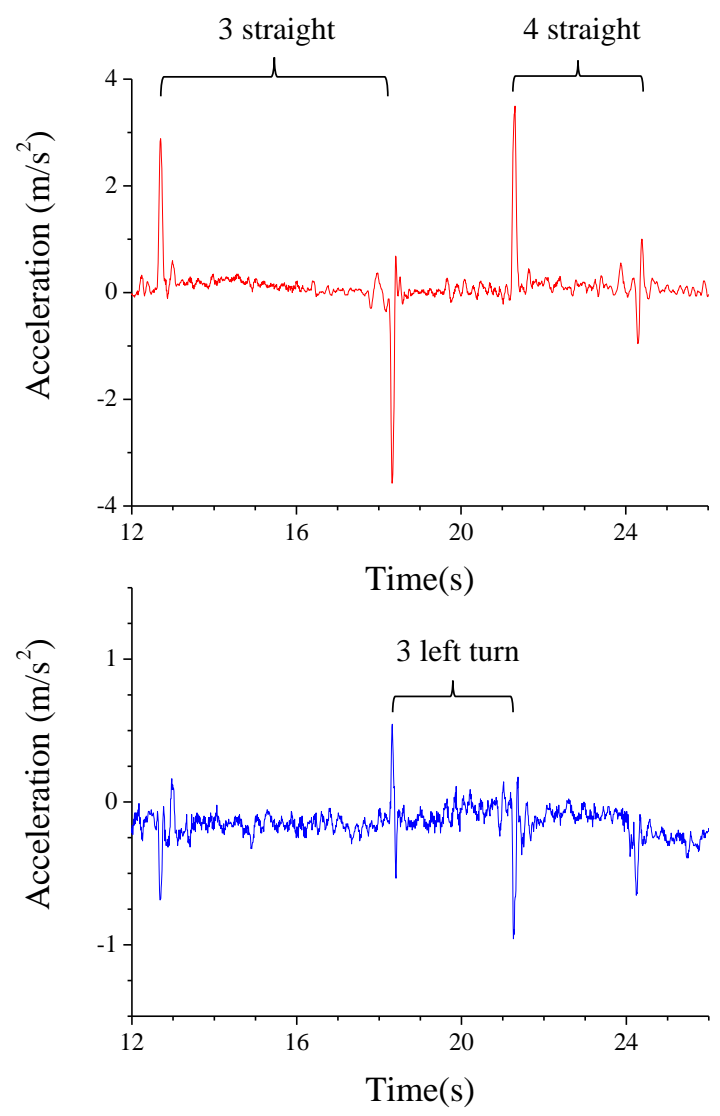

Fig.10 Detail of the measured acceleration (12 to 26 second) (1) Pitch acceleration (2) yaw acceleration
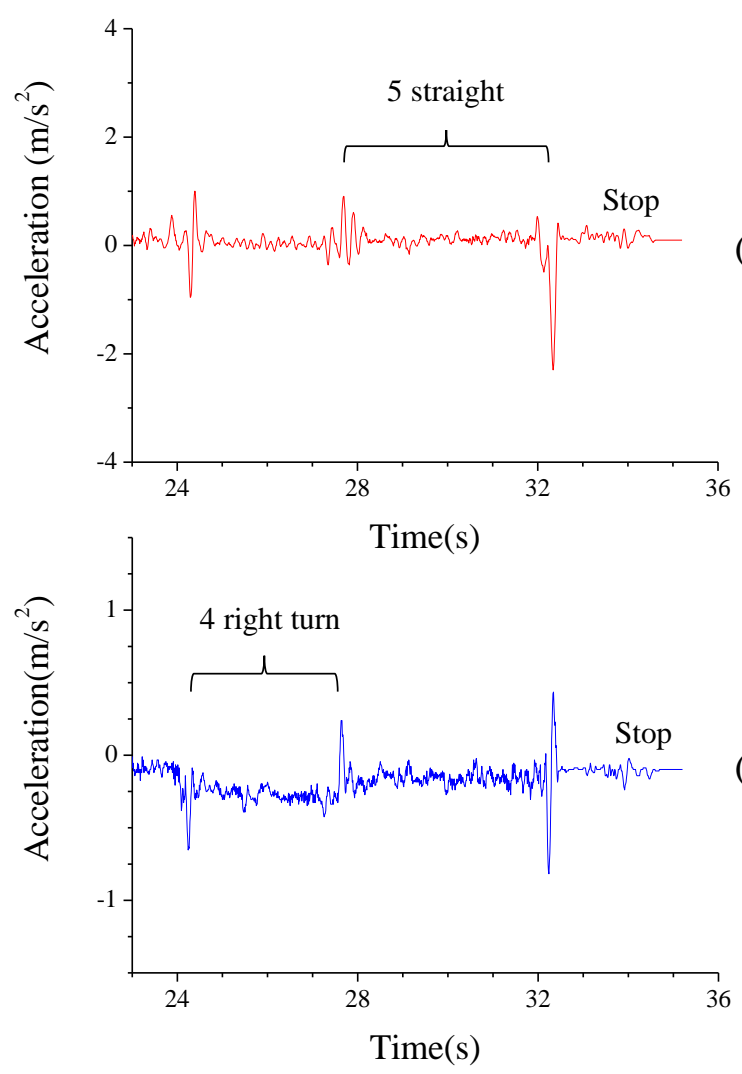

Fig.11 Detail of the measured acceleration (23 to 36 second) (1) Pitch acceleration (2) yaw acceleration

\section{Conclusion}

We designed and fabricated a PMV that has the potential to be used as a novel transportation device for disabled and elderly persons and considered its utilization. The myoelectric potential suitable for PMV control was investigated. The relationship between grip strength and myoelectric potential was tested for both the common digital extensor muscle and the deep digital flexor muscle, and the proportional relationship between grip strength and myoelectric potential was confirmed. We proposed two myoelectric potential commands to control the PMV and utilized hysteresis as a criterion for judgment. We evaluated continuous operation by employing the myoelectric potential commands by driving on a simple test course. According to the experimental results, the PMV could be controlled according to the driver's intentions, but had some shortcomings. The reason for these were considered as follows. First, the time between the initiation and myoelectric potential of the driver is not constant, and the amplitude of the generated myoelectric potential was not stable. For this reason, the PMV sometimes responds with a slight delay from the time of the driver's intention. Next, it is conceivable that a difference of several tens of centimeters occurs depending on the position of the PMV passing through the course because the PMV is $350 \times 500$ $\mathrm{mm}$. Last, the revolution speed of the DC motor changes owing to the unstable torque required by the condition of the course because the motor drive system does not use feedback control. These concerns should be considered and improved upon in the future. However, it was confirmed that the PMV could be controlled mostly along with the driver's intentions. These experimental results imply the feasibility of a PMV operated by myoelectric potential.

\section{References}

(1) N.Hashimoto, Research Status and Challenges of Mobility Robot as New Mobility, Institute of Electronics, Information, and Communication Engineers, Vol.97, No.6, pp.507-512, 2014.

(2) N.Akai, T.MATSUDA, K.INOUE, R.UNAI, K.YAMAUCHI, J.YAMAMOTO, K.OZAKI, Development of a Personal Mobility Robot to Collect Magnetic Data in Large-scale Environment,

(3) R.TANI, Y.HIRAKI, T.YAMASHITA, S.SHIMODA, Verifying the Effectiveness of Personal Mobility Vehicle "STAVi" in Schools, 
(4) T.MATSUDA, K.INOUE, N.AKAI, M.NAKADA, K.OZAKI, Conceptual Design of Personal Mobility and Challenge of Development for Special Zone of Experiment by Mobility Robots in Tsukuba,

(5) T.Yoshimura, and M.Okugawa, Design of Seating Wheeled Inverted Pendulum Type Personal Mobility,

(6) M.MIURA, Y.TAKAHASHI, Proposal for Personal Mobility Vehicle for People with Limited Mobility,

(7) http://www.cybathlon.ethz.ch/en/

(8) S.Matsugaura, H.Nishimura, M.Omae, and H.Shimizu, "Development of a Driver-monitoring Vehicle Based on an Ultra Small Electric Vehicle", Journal of Asian Electric Vehicle, Vol.3, No.2, pp.757-762, 2005.
(9) C.Zhu, Y.Okada, M.Yoshiokak, T.Yamamoto, H.Yu, Y.Yan, "Power Augumentation of Upper Extremity by Using Agonist Electromyography Signal Only for Extended Admittance Control", Journal of Industry Applications, Vol.3, No.3, pp.260-269, 2014.

(10) Y.Iwamoto, D.Umetsu, and S.Ozaki, "Evaluation of Driver-vehicle Matching using Neck Muscle Activity and Vehicle Dynamic", Kansei Engineering International Journal, Vol.11, No.3, pp.147-154, 2012.

(11) Makikawa, Yoshida, Nambu, Shiozawa, Okada, "Measurement technologies of mind and body condition in daily life for the development of human friendly products", Corona publishing co. 1td, 2010, in Japanese. 